\title{
Non-visual GRKs: are we seeing the whole picture?
}

\author{
Jonathon M. Willets, R.A. John Challiss and Stefan R. Nahorski
}

Department of Cell Physiology \& Pharmacology, University of Leicester, Maurice Shock Medical Sciences Building, University Road, Leicester LE1 9HN, UK

\begin{abstract}
G-protein-coupled receptor kinases (GRKs) comprise a family of seven mammalian serine/threonine protein kinases that phosphorylate and regulate agonist-occupied or constitutively active G-protein-coupled receptors (GPCRs). Studies of the details and consequences of these mechanisms have focused heavily on the original $\beta$-adrenoceptor kinase ( $\beta$-ARK) family (GRK2 and GRK3) and, in particular, on phosphorylation-dependent recruitment of adaptor proteins such as the $\beta$-arrestins. However, recent work has indicated roles for the other, non-visual GRKs (GRK4, GRK5 and GRK6) and has revealed potential phosphorylation-independent regulation of GPCRs by GRK2 and GRK3. In this article, we review this newer information and attempt to put it into context with GRKs as physiological regulators that could be appropriate targets for future pharmacological intervention.
\end{abstract}

G-protein-coupled receptors (GPCRs) constitute a very large family of heptahelical, integral membrane proteins that mediate a wide variety of physiological processes ranging from the transmission of light and odorant signals to the mediation of neurotransmission and hormonal actions [1,2]. GPCRs represent a major target for therapeutic agents, and the continuing identification of orphan GPCRs offers opportunity for future pharmacological and therapeutic development [3].

Most GPCRs display a rapid loss of responsiveness in the continuing (or recurring) presence of an agonist or stimulus, and there is now substantial evidence that this process of desensitization, at least in part, is a consequence of ligand-induced phosphorylation of serine and threonine residues located within the $\mathrm{C}$-terminal domain and/or the third intracellular loop of GPCRs. Two families of protein kinases appear to be predominantly involved: the G-protein-coupled receptor kinases (GRKs), which phosphorylate agonist-occupied GPCRs to mediate homologous receptor phosphorylation, and the second messengeractivated kinases, such as cAMP-dependent kinase (PKA) and protein kinase C (PKC), which can phosphorylate both ligand-bound and other inactive GPCRs in a heterologous manner $[4,5]$. Phosphorylation by GRKs enhances receptor affinity for non-visual $\beta$-arrestins 1 and 2 (arrestin2 and arrestin3), which not only sterically suppresses further interaction between the receptor and

\footnotetext{
Corresponding author: Stefan R. Nahorski (srn@leicester.ac.uk).
}

G proteins, but also initiates clathrin-mediated endocytosis of phosphorylated receptors and can promote the activation of additional signalling pathways by acting as agonist-regulated adaptor scaffolds [6].

Other protein kinases have also been implicated in GPCR regulation. For example, evidence has accumulated that casein kinase $1 \alpha$ might bring about agonistmediated phosphorylation of acetylcholine muscarinic $\mathrm{M}_{3}$ receptors and light-activated rhodopsin [7]. Casein kinase $1 \alpha$-mediated phosphorylation does not appear to be associated with reduced responsiveness of the $M_{3}$ receptor but probably contributes to the activation of extracellular signal-regulated kinases 1 and $2($ ERK1,2) by this receptor $[8,9]$.

Although research in this area has provided some remarkable insights into the mechanisms of GPCR regulation and revealed new information on the longestablished phenomenon of drug-induced desensitization (tachyphylaxis), many issues remain unresolved. Almost all of the mechanisms described have been identified following research on the intensely studied GRK2 and GRK3 family [originally known as $\beta$-adrenoceptor kinase 1 and 2 ( $\beta$-ARK1,2)] and it has been widely assumed (often without evidence) that common mechanisms apply to GRK4, GRK5 and GRK6. It is becoming increasingly clear that these GRKs display different tissue distributions, subcellular localization and regulation of their intrinsic kinase activity $[10,11]$. However, it is not clear whether these kinases show discrete specificity for groups of GPCRs in vivo or whether their localization and regulation allows a more coordinated regulation in concert with other kinases. In this respect it is also important to stress that there is substantial evidence that phosphorylation of GPCRs is not always associated with desensitization [12-14] and that GRKs can regulate GPCR signalling in a phosphorylation-independent manner (Box 1). Finally, receptor desensitization traditionally requires receptor phosphorylation before internalization. However, growing evidence suggests that these processes can occur independently [15-17]. Thus, it is conceivable that GRKs 4-6 mediate phosphorylations that affect the desensitization and/or internalization of specific GPCRs. GPCR internalization and trafficking have been extensively reviewed recently [18] and, with this in mind, we focus here specifically on the role of GRKs in the acute phosphorylation-dependent (and phosphorylationindependent) desensitization phase of GPCR regulation. 


\section{Box 1. GRKs can regulate GPCRs in a phosphorylation-independent manner}

Recent studies have provided evidence that certain G-protein-coupled receptor kinases (GRKs) can suppress the interaction between G-proteincoupled receptors (GPCRs) and G proteins in a phosphorylationindependent manner. Initial surprises came from work on the $\alpha_{1 \mathrm{~B}}$-adrenoceptor [71] and the endothelin $\mathrm{ET}_{\mathrm{A}}$ and $\mathrm{ET}_{\mathrm{B}}$ receptors [72], which demonstrated that overexpression of either wild-type GRK2 or a catalytically inactive mutant form of this protein suppressed receptorstimulated $\mathrm{G}_{\mathrm{q} / 11}$-mediated phospholipase C (PLC) activation. This has now been observed with several other PLC-linked GPCRs in a variety of cell backgrounds $[24,28,37,73]$. Although these phosphorylationindependent actions of GRK2 were initially attributed to the ability of GRK2 to sequester G $\beta \gamma$-subunits [67], it now seems more likely that GRK2 selectively binds to the activated form of $\mathrm{G} \alpha_{\mathrm{q} / 11}$ (although not to $\mathrm{G} \alpha_{\mathrm{s}}, \mathrm{G} \alpha_{\mathrm{i}}$ or $\left.\mathrm{G} \alpha_{12 / 13}\right)$ to suppress PLC- $\beta$ activation.

In a key study, Carman et al. [22] revealed that although the $\mathrm{N}$-terminal regulator of G-protein signalling (RGS)-like domains of GRK2 and GRK3 (see Figures 1 and 2 in the main text) possess only modest GTPaseactivating protein (GAP) activity, they bind avidly to activated $\mathrm{G} \alpha_{\mathrm{q} / 11}$ to inhibit inositol $(1,4,5)$-trisphosphate $\left[\operatorname{lns}(1,4,5) P_{3}\right]$ generation. Crucially, the expression of this RGS-like domain alone can inhibit the ability of several GPCRs to stimulate PLC activity, while exerting no effect on cAMP production [23]. Although the N-terminals of all GRKs possess sequences that are homologous to the RGS family, only GRK2 and GRK3 exhibit this strong interaction with $\mathrm{G}_{\mathrm{q} / 11}$. Recent studies using mutations in the RGS homology domain have revealed a novel sequence, present in only GRK2 and GRK3 (the 'C-site' [53]), that avidly binds to $G \alpha_{q / 11}$. This interaction is different from those of other RGS

\section{GRK structure and distribution}

There are three sub-groups within the GRK family. GRK1 (rhodopsin kinase) and GRK7 (cone opsin kinase) form one distinct sub-group that is only found in retinal cells. The non-visual GRKs divide into two sub-groups: the GRK2 subfamily, consisting of GRK2 ( $\beta$-ARK1) and GRK3 ( $\beta$-ARK2), and the GRK4 subfamily, consisting of GRK4, GRK5 and GRK6. GRK4 is predominantly found in the testes [19] and, to lesser extent, in some brain regions and the kidney [20,21], whereas GRKs 2, 3, 5 and 6 are widely expressed (Table 1).

The basic structure of non-visual GRK family members is similar (Figure 1), with a highly conserved central (263-266 amino acid) catalytic domain. The N-terminal 185 amino acid region displays considerable homology between individual GRKs. The similarity of the N-termini of GRKs has led to speculation that this region might be important in receptor recognition. All non-visual GRKs have a regulator of G-protein signalling (RGS) domain proteins and maps almost exclusively to the $\alpha 5$ helix of the RGS-like domain of GRK2 [53].

The unique and potent interactions of GRK2 and GRK3 with $G \alpha_{q / 11}$ raise the question of their significance: that is, do these GRKs act principally as C-site RGS-like proteins in vivo? A criticism that can be raised is whether such effects are a consequence of overexpression of GRK2 or GRK2 mutants. Suppression of endogenous GRK expression in primary cells using an antisense strategy or RNA interference approach might help address this important issue. Indeed, Perroy et al. [30] using a short interfering RNA (siRNA) strategy have recently provided evidence that GRK4 might also regulate $\mathrm{GABA}_{B}$ receptors in a phosphorylationindependent manner. Whether this action results from binding to $\mathrm{G} \alpha_{\mathrm{i} / \mathrm{o}}$ or the receptor remains to be established. Earlier studies on endothelin [72] and parathyroid hormone [74] receptor signalling established that GRK2 can interact directly with the receptor, probably by an interaction with the $\mathrm{N}$-terminus of GRK2. Indeed, Dhami et al. [24] have demonstrated that an $\mathrm{N}$-terminal polypeptide of GRK2 $\left({ }^{\Delta 35-185} \mathrm{GRK} 2\right)$ co-immunoprecipitates with the type 1 metabotropic glutamate receptor and suppresses its signalling potential. This suggests that, in addition to their interaction with $\mathrm{G} \alpha_{\mathrm{q} / 11}$, GRK2 and GRK3 (and perhaps GRK4) might also interact with GPCRs, but again emphasizes a phosphorylation-independent regulation of GPCRs. An investigation [57] of the crystallographic structure of bovine GRK2 complexed with G $\beta \gamma$-subunits has revealed that the kinase can bind the GPCR and $G \beta \gamma$ - and $G \alpha_{q / 11}$-subunits simultaneously and the authors of this study suggested that this might acutely attenuate signalling while kinase-mediated phosphorylation and recruitment of arrestins is being completed to terminate signalling.

within the N-terminus region (Figure 1), which provides a potential mechanism by which GRKs might regulate GPCR signalling via phosphorylation-independent mechanisms. Indeed, growing evidence suggests that this could be the case for GRK2 and GRK3 [22-24] (Box 1). GRK4-6 possess a highly conserved binding site (amino acids 22-29 for GRK5) for phosphatidylinositol (4,5)-bisphosphate $\left[\operatorname{PtdIns}(4,5) P_{2}\right]$, which is thought to enhance catalytic activity [10].

The C-terminal of GRK2 and GRK3 is longer than that of the GRK4 subfamily, and contains a 125 amino acid pleckstrin homology $(\mathrm{PH})$ domain. This domain contains binding sites for both PtdIns $(4,5) P_{2}$ and free G $\beta \gamma$, which play a role in targeting and translocation of these primarily cytosolic GRKs to membranes following GPCR activation [10]. More recently, a second binding site for G $\beta \gamma$-subunits has been identified within the first 53 amino acids of GRK2 [25], which suggests that either the N- or the C-terminal regions might be sufficient to allow GRK2

Table 1. Tissue distribution of GRKs ${ }^{a-c}$

\begin{tabular}{|c|c|c|c|c|c|c|}
\hline Tissue & GRK2 & GRK3 & GRK4 & GRK5 & GRK6 & Refs \\
\hline Brain & $\begin{array}{l}+(\mathrm{mRNA} \text { and protein by } \\
\text { WB) }\end{array}$ & + & $+($ Purkinje cells $)$ & $\begin{array}{l}+(\text { mRNA and protein by } \\
\text { WB) }\end{array}$ & $\begin{array}{l}+(\mathrm{mRNA} \text { and protein by } \\
\text { WB) }\end{array}$ & {$[61,64]$} \\
\hline Heart & $\begin{array}{l}+(\mathrm{mRNA} \text { and protein by } \\
\text { WB) }\end{array}$ & & & + & & {$[61]$} \\
\hline Haematopoietic & $\begin{array}{l}+(\text { mRNA and protein by } \\
\text { WB })\end{array}$ & & & $+(\mathrm{WB})$ & $\begin{array}{l}+\underset{\text { WB }}{(\mathrm{mRNA}} \text { and protein by } \\
\end{array}$ & {$[62,65]$} \\
\hline Lungs & & & + & & + & {$[61]$} \\
\hline Kidney & & & + & + & & {$[29,61]$} \\
\hline Skeletal muscle & + & $+($ ELISA $)$ & & & + & [61] \\
\hline Testes & & & + & & & [61] \\
\hline Olfactory & & + & & & & [63] \\
\hline
\end{tabular}

${ }^{a}$ GRKs $2,3,5$ and 6 are thought to be expressed ubiquitously throughout all tissues; however, the data shown indicate studies that identify tissues in which individual GRKs are highly expressed.

bGRK mRNA was detected, unless otherwise indicated.

'Abbreviations: ELISA, enzyme-linked immunosorbent assay; GRK, G-protein-coupled receptor kinase; WB, western blot. 


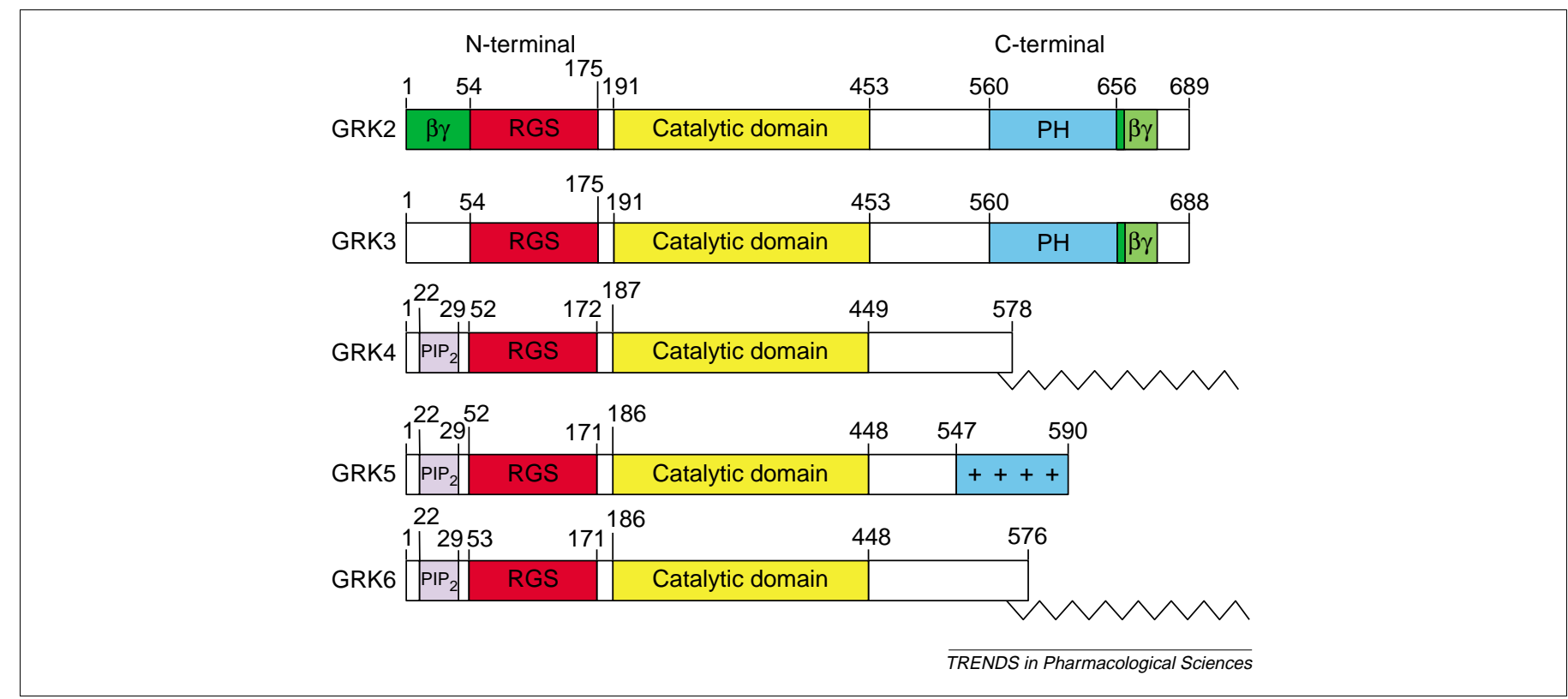

Figure 1. The structure of non-visual G-protein-coupled receptor kinases (GRKs). All GRKs possess a conserved N-terminal regulator of G-protein signalling (RGS)-like domain [22], the relevance of which is discussed in Box 1. In addition, all GRKs have a highly conserved central serine/threonine kinase catalytic domain. The major differences between members of the GRK subfamilies occur within the $\mathrm{N}$ - and $\mathrm{C}$-terminal domains. The GRK2 and GRK3 subfamily are primarily cytosolic proteins that are recruited to the plasma membrane following activation of receptors by agonists, a process that is aided by two specific domains at either end of the protein. The $\mathrm{C}$-terminal pleckstrin homology $(\mathrm{PH})$ domain contains sites for $\mathrm{G} \beta \gamma$ and phosphatidylinositol $(4,5)$-bisphosphate $\left(\mathrm{PIP}_{2}\right)$ binding [10]. More recently, the N-terminal RGS-like domain has been shown to bind to activated GTP-bound $\mathrm{G} \alpha_{\mathrm{q} / 11}$ [22], which not only blocks the interaction of $\mathrm{G} \alpha_{\mathrm{q} / 11}$ with phospholipase $\mathrm{C}$ (PLC), but might also assist the correct targeting of GRK2 and GRK3 before receptor phosphorylation [57]. A second G $\beta \gamma$ binding site has recently been mapped to the N-terminus of GRK2 [25] (dark green), which, given the almost complete sequence homology between the two kinases in this region, also suggests a similar site for GRK3. The GRK4-6 subfamily is predominantly membrane associated. For GRKs 4 and 6 this is achieved via palmitoylation at cysteine residues within the C-terminal region [10]. GRK5 contains a polybasic region of 43 amino acids (547-590) [26], which interacts with negatively charged plasma membrane lipids. In addition, GRKs 4,5 and 6 possess a PIP 2 binding region within the $\mathrm{N}$-terminus, which is also thought to assist in membrane association. Amino acid numbering is given for the human GRK family members.

targeting to the membrane. GRK4 and GRK6 are posttranslationally palmitoylated at one or more cysteine residues clustered within the last $15-20$ amino acids of the C-terminus, leading to an exclusive membrane-associated localization [10]. GRK5 is also predominantly membrane associated, and in this case localization is not achieved through lipidation but instead through the $\operatorname{PtdIns}(4,5) P_{2}$ binding domain of the $\mathrm{N}$-terminus and a polybasic region (amino acids 547-560) close to the C-terminus [26]. Further heterogeneity is possible within the GRK4 subfamily because both GRK4 and GRK6 are expressed in multiple splice variant forms [27]. Indeed, one splice variant of GRK4 lacks the N-terminal $\operatorname{PtdIns}(4,5) P_{2}$ binding region, although the physiological significance of isoformic variation is not understood at present. GRKs 1 and 7 share many structural similarities with the nonvisual GRKs, including an N-terminal RGS-like domain and central catalytic domain. Both GRK1 and GRK7 are membrane associated; however, unlike GRKs 4 and 6, this association is via post-translational farnesylation at the C-terminal.

\section{Experimental approaches}

In many studies the involvement of various GRKs in the regulation of GPCRs is often implied by overexpression of both receptor and kinases, usually in heterologous expression systems. Although this approach indicates that certain GPCRs can be substrates for several GRKs, it is limited because it fails to determine whether endogenously expressed GRKs regulate GPCR function. With this in mind, we focus here on studies in which manipulation of the activity of endogenous GRKs is the adopted strategy. In a large number of studies this has been achieved by the overexpression of 'catalytically dead' dominant-negative GRK constructs and this has led to important conclusions regarding endogenous kinases. However, it should be noted that this too is an overexpression approach and, in addition to competing with endogenous kinases, such mutants might also bind to receptors or other regulatory proteins. Thus, their use should be undertaken with caution and, where possible, in conjunction with alternative (e.g. antisense and/or RNA interference) approaches.

\section{What do we know about endogenous GRK4, GRK5 and GRK6 function?}

\section{GRK4}

Because of its limited distribution (Table 1) GRK4 has tended to be overlooked as a kinase that might regulate GPCRs other than those expressed in the testes. However, GRK4 appears to play a specific role in the regulation of GPCRs in tissues in which its expression is relatively high (e.g. cerebellar Purkinje cells [21,28] and the renal medulla [20]). Studies using antisense oligonucleotides to reduce selectively the expression of GRK2 or GRK4 in rat Purkinje cells or human renal proximal tubule cells have revealed key differences between these kinases. In Purkinje cells, loss of GRK4, but not loss of GRK2, inhibited type 1a metabotropic glutamate $\left(\mathrm{mGlu}_{1 \mathrm{a}}\right)$ receptor desensitization [21]. In addition, reduction of GRK4 expression in renal proximal tubular cells produced a 
concurrent reduction of dopamine D1 receptor desensitization, whereas loss of GRK2 in this tissue had no effect on such desensitization [29]. Very recent studies [30] that used a short interfering RNA (siRNA) approach to decrease GRK4 activity has provided strong evidence for a specific role of GRK4 in the desensitization of $\mathrm{GABA}_{\mathrm{B}}$ receptors in cerebellar granule cells. Moreover, this effect appeared to be mediated independently of kinase activity. A transgenic mouse overexpressing the polymorphism A142V GRK4 has been reported to display a hypertensive phenotype that is associated with impaired $\mathrm{Na}^{+}$excretion [31]; however, the knockout GRK4 $4^{-l-}$ phenotype has not yet been described.

\section{GRK5}

Despite several studies that have shown that GRK5 is able to phosphorylate many GPCRs when coexpressed in heterologous systems, relatively few studies have examined either endogenously expressed receptors or specific inhibition of endogenous GRK5 function. Shetzline and co-workers found little selectivity between GRKs for vasoactive intestinal polypeptide type $1\left(\mathrm{VPAC}_{1}\right)$ receptor phosphorylation and desensitization when GRK and receptors were coexpressed in HEK293 cells [32]. However, only GRK5 and GRK6 were able to desensitize endogenously expressed $\mathrm{VPAC}_{1}$ receptors. Endogenous GRK5 also appears to play an important role in the regulation of thrombin-activated signalling in human dermal microvascular endothelial cells [33]. Overexpression of wild-type GRK5 decreased thrombin-induced phosphoinositide hydrolysis and intracellular $\mathrm{Ca}^{2+}$ responses, whereas introduction of a dominant-negative GRK5 enhanced thrombin-induced $\mathrm{Ca}^{2+}$ signalling. GRK2, GRK6 and dominant-negative GRK2 were ineffective. Using full-length antisense constructs for GRK5 and GRK6 stably expressed in FRTL5 rat thyroid cells, Nagayama et al. [34] showed a selective inhibition of thyrotropin receptor desensitization in GRK5-deficient, but not GRK6-deficient, cells.

Initial studies from GRK5 ${ }^{-/-}$knockout mice showed an enhanced sensitivity to the central administration of the muscarinic receptor agonist oxotremorine [35]. The reported behavioural changes associated with GRK5 knockout, enhanced hypothermia and hypoactivity, suggest that central muscarinic receptors are regulated by endogenous GRK5. This interaction appeared to be specific because $\mathrm{D} 1$ or $5 \mathrm{HT}_{1 \mathrm{~A}}$ receptor regulation were unaffected by GRK5 ablation [35].

\section{GRK6}

Growing evidence highlights the potential role of endogenous GRK6 in GPCR regulation. Lazari et al. [36] have shown that inhibition of endogenous GRK2 or GRK6, via the introduction of dominant-negative kinase constructs, blocked rat follitrophin receptor phosphorylation equally in HEK293 cells. However, only inhibition of endogenous GRK2 led to inhibition of follitrophin receptor internalization. When expressed in HEK293 cells depleted of GRKs 2, 5 or 6 through the use of antisense oligonucleotides, the calcitonin gene-related peptide receptor appeared to be specifically regulated by endogenously expressed GRK6 [37].

As stated above, when overexpressed many GRKs are able to phosphorylate GPCRs in an agonist-dependent manner. Indeed, we recently showed that stable overexpression of either GRK3 or GRK6 is able to enhance phosphorylation of the endogenously expressed muscarinic $\mathrm{M}_{3}$ receptor in SH-SY5Y neuroblastoma cells [38]. However, only GRK6-mediated phosphorylation was accompanied by enhanced receptor-G-protein uncoupling. Furthermore, inhibition of endogenously expressed GRK6 via the stable expression of the dominant-negative kinase $\left({ }^{\mathrm{K} 215 \mathrm{R}} \mathrm{GRK} 6\right)$ led to a $50 \%$ inhibition of both agoniststimulated $\mathrm{M}_{3}$ receptor phosphorylation and subsequent receptor-G protein uncoupling [39]. These data suggest that GPCR-GRK interactions might prove to be highly specific when examining the regulation of endogenously expressed receptors. Thus, recent studies have revealed that overexpression of dominant-negative GRK6 in NG108-15 mouse neuroblastoma $\mathrm{x}$ rat glioma cells blocked the desensitization of endogenous secretin receptors [40], whereas dominant-negative GRK5 had no effect. By contrast, knockout of up to $70 \%$ of GRK2 using an antisense strategy in the same cells had no effect on secretin receptor responsiveness but selectively enhanced adenosine $\mathrm{A}_{2}$ receptor signalling [41]. Finally, very recent observations that GRK6 rather than GRK2 initiates homologous $\beta$-adrenoceptor desensitization in uterine smooth muscle cells [42] highlight that this GPCR can be a target for different GRKs depending on the tissue and perhaps the physiological status.

Further strong evidence for the involvement of GRK6 in the regulation of GPCR signalling has come from studies using GRK knockout mice. Comparison of the chemotactic responses of $\mathrm{T}$ and $\mathrm{B}$ cells isolated from $\mathrm{GRK} 5^{-/-}$or $\mathrm{GRK}^{-/-}$mice indicated that GRK6-deficient cells were impaired in their ability to respond to the chemokine CXCL12 (a selective ligand for the chemokine receptor CXCR4), whereas GRK5-deficient cells were unaffected [43]. The potential role of GRK6 in dopamine receptor regulation has also been highlighted recently in GRK6 $6^{-/-}$ mice, which showed supersensitivity to psychostimulant drugs [44]. Thus, cocaine- and amphetamine-induced locomotor activity was enhanced in $\mathrm{GRK} 6^{-/-}$mice compared with wild-type animals, despite unaltered dopamine homeostasis. These data, together with evidence for an enhanced coupling of dopamine D2 and D3 receptors to $\mathrm{G}_{\mathrm{i} / \mathrm{o}}$ proteins in $\mathrm{GRK} 6^{-/-}$mice, suggest that GRK6 regulates postsynaptic D2 and/or D3 receptors.

\section{Does the regulation of non-visual GRKs provide clues to their specificity?}

Although it is well established that agonist activation of GPCRs is a prerequisite for GRK-mediated phosphorylation, relatively little is known about the exact recruitment signals for individual GRKs. The simplest model that can be proposed is one where the conformational change following agonist binding exposes domains of the receptor to 'random' kinase phosphorylation and the kinase(s) responsible will be determined by kinase availability and localization $[10,45]$. However, this model seems unlikely to 
Table 2. Regulation of non-visual GRK activities ${ }^{a}$

\begin{tabular}{|c|c|c|c|c|}
\hline Regulator & GRK & Effect & Region of GRK involved & Refs \\
\hline$\alpha$-Actinin & All GRKs & Inhibits catalytic activity & N-terminal & {$[66]$} \\
\hline $\mathrm{Ca}^{2+}$-calmodulin & GRKs 2-6 & $\begin{array}{l}\text { Inhibits GRK activity; potency } \text { IC }_{50} \text { : } \\
\text { GRK5 }(40 \mathrm{nM})>\text { GRK4 }(80 \mathrm{nM})>\text { GRK6 }> \\
\text { GRK2 }(2 \mu \mathrm{M})\end{array}$ & $\begin{array}{l}\text { For GRK5 N-terminal (aa } 20-39 \text { ) } \\
\text { and C-terminal (aa 540-578) }\end{array}$ & {$[48,60]$} \\
\hline Caveolin & Probably all & Inhibits catalytic activity & N-terminal & {$[46]$} \\
\hline $\mathrm{G} \alpha_{\mathrm{q} / 11}$ & GRK2, GRK3 & $\begin{array}{l}\text { Inhibits PLC signalling via physical binding } \\
\text { to GTP-bound } G \alpha\end{array}$ & RGS domain on $\mathrm{N}$-terminal & {$[22,23]$} \\
\hline $\mathrm{G} \beta \gamma$ & GRK2, GRK3 & $\begin{array}{l}\text { Required for membrane association and } \\
\text { GPCR targeting }\end{array}$ & $\begin{array}{l}\text { N-terminal (aa 1-53) and C-terminal } \\
\text { PH domain (aa 643-670) }\end{array}$ & {$[25,67]$} \\
\hline Ptdlns $(4,5) P_{2}$ & All GRKs & $\begin{array}{l}\text { Stimulates catalytic activity and promotes } \\
\text { membrane association }\end{array}$ & $\begin{array}{l}\text { C-terminal for GRK2, and GRK3 PH } \\
\text { domain; N-terminal for GRKs } 4,5 \text { and } 6 \\
\text { (aa 22-29) }\end{array}$ & [10] \\
\hline PtdSer & GRK2 & Stimulates kinase actvity & C-terminal probably distal to $\mathrm{PH}$ domain & {$[10]$} \\
\hline PKC & GRK2, GRK5 & $\begin{array}{l}\text { Promotes GRK2 membrane recruitment; } \\
\text { inhibits GRK5 activity }\end{array}$ & $\begin{array}{l}\text { GRK2 Ser29; N-terminal GRK5 (within } \\
\text { aa 571-590) }\end{array}$ & {$[58,68,69]$} \\
\hline MAPK (ERK) & GRK2 & Inhibits catalytic activity & Serine 670 & {$[55]$} \\
\hline c-Src & GRK2 & $\begin{array}{l}\text { Stimulates catalytic activity and enhances } \\
\text { GRK2 degradation }\end{array}$ & N-terminus (aa $13,86,92$ ) & {$[59,70]$} \\
\hline
\end{tabular}

${ }^{a}$ Abbreviations: aa, amino acid; ERK, extracellular signal-regulated kinase; GPCR, G-protein-coupled receptor; GRK, G-protein-coupled receptor kinase; MAPK, mitogenactivated protein kinase; PH, pleckstrin homology; PKC, protein kinase C; PLC, phospholipase C; Ptdlns $(4,5) P_{2}$, phosphatidylinositol $(4,5)$-bisphosphate; PtdSer, phosphatidylserine; RGS, regulator of G-protein signaling; Src, non-receptor tyrosine kinase Src.

describe the major physiological mechanism because other receptor domains exposed on agonist binding appear to promote GRK-receptor interactions independently of regions that are subsequently phosphorylated [10,45]. Despite the lack of information regarding the initiation of GPCR and GRK interactions, many positive and negative regulators of GRK activity have been identified and reviewed extensively $[10,45,46]$. Thus, a diverse array of proteins and lipids can regulate GRK activity, either affecting membrane localization or enzymatic activity as a result of GRK phosphorylation by a variety of kinases. Some of these interactions are shown in Table 2 and Figure 2.

In the case of GPCRs that couple to PLC- $\beta$ via $\mathrm{G} \alpha_{\mathrm{q} / 11}$ or G $\beta \gamma$, an array of regulatory features could determine the overall regulation of signalling by different GRKs (Figure 2). Activators of these GPCRs can dramatically deplete membrane PtdIns $(4,5) P_{2}$ levels within seconds of agonist addition [47], raise the intracellular concentration of $\mathrm{Ca}^{2+}$ many-fold, and recruit and activate several PKC isoenzymes through increases in the levels of diacylglycerol and/or $\mathrm{Ca}^{2+}$ Membrane association of the different GRKs is regulated by multiple factors (e.g. covalent lipidation and G $\beta \gamma$ binding), and it is therefore possible that local or global PtdIns $(4,5) P_{2}$ depletion might differentially affect the anchoring of the GRK2 and GRK3 proteins at the plasma membrane compared with members of the GRK4 subfamily [10]. Likewise, activation of PKC might be anticipated to enhance GRK2 activity but diminish the activity of GRK5 towards GPCR phosphorylation, and the relative affinity of $\mathrm{Ca}^{2+}$-calmodulin for the different GRKs [48] also highlights the potential for differential regulation of the non-visual GRKs. Collectively, therefore, one might anticipate that if multiple GRKs are coexpressed in certain tissues, GRK2, but not GRK5, might be a favoured regulator of $\mathrm{G} \alpha_{\mathrm{q} / 11}-\mathrm{PLC}$-coupled GPCRs. However, the reality of this in functioning intact cells or within tissues in vivo lacks evidence at present. Indeed, Budd et al. [49] failed to observe an alteration in agonist-mediated phosphorylation of the $\beta_{2}$-adrenoceptor in Chinese hamster ovary $(\mathrm{CHO})$ cells, despite dramatic alterations in $\operatorname{PtdIns}(4,5) P_{2}$ and $\mathrm{Ca}^{2+}$ concentrations mediated by $\mathrm{M}_{3}$ receptor co-activation. It will be important for these studies to be extended to cells in which GRKs 4,5 and 6 are known to be active in modifying GPCR functions.

The complex nature of kinase-mediated receptor phosphorylation is highlighted in studies that have attempted to determine the individual phospho-acceptor sites involved. Three serine residues were identified in the C-terminal tail of the bradykinin $\mathrm{B}_{2}$ receptor as principal phospho-acceptor sites [13]. Despite the lack of apparent selectivity between GRKs, phosphopeptide mapping identified distinct phosphorylation profiles between different GRKs, suggesting the possibility of differential regulation. More recently, an elegant study using phospho-specific antibodies directed against four individual serine-containing epitopes within the C-terminal tail of the chemokine receptor CCR5 highlighted the distinct stimulus strength and temporal patterns of PKC- and GRK-mediated phosphorylation [50]. Overall, there is increasing evidence for specificity of GRK-GPCR interactions when endogenous kinases are investigated in physiological settings. As more is revealed about the differential regulation and/or recruitment of different GRKs and the specific patterns of receptor phosphorylation, it would seem certain there will be a renewed appreciation of the specificity of the non-visual GRKs.

Potential therapeutic targets for selective GRK inhibition Despite the use of heparin, $\mathrm{Zn}^{2+}$ and suramin as nonselective inhibitors directed at the ATP binding site, at present there are no specific inhibitors of individual GRK activity. This is perhaps surprising because GRK expression levels are known to change in several disease states [10], and could be considered to be logical molecular targets with respect to the pharmacological manipulation of GPCR responsiveness. Furthermore, there are several distinct structural differences between the GRK subfamilies that could be exploited for therapeutic intervention. However, approaches using subtype-specific antibodies or 


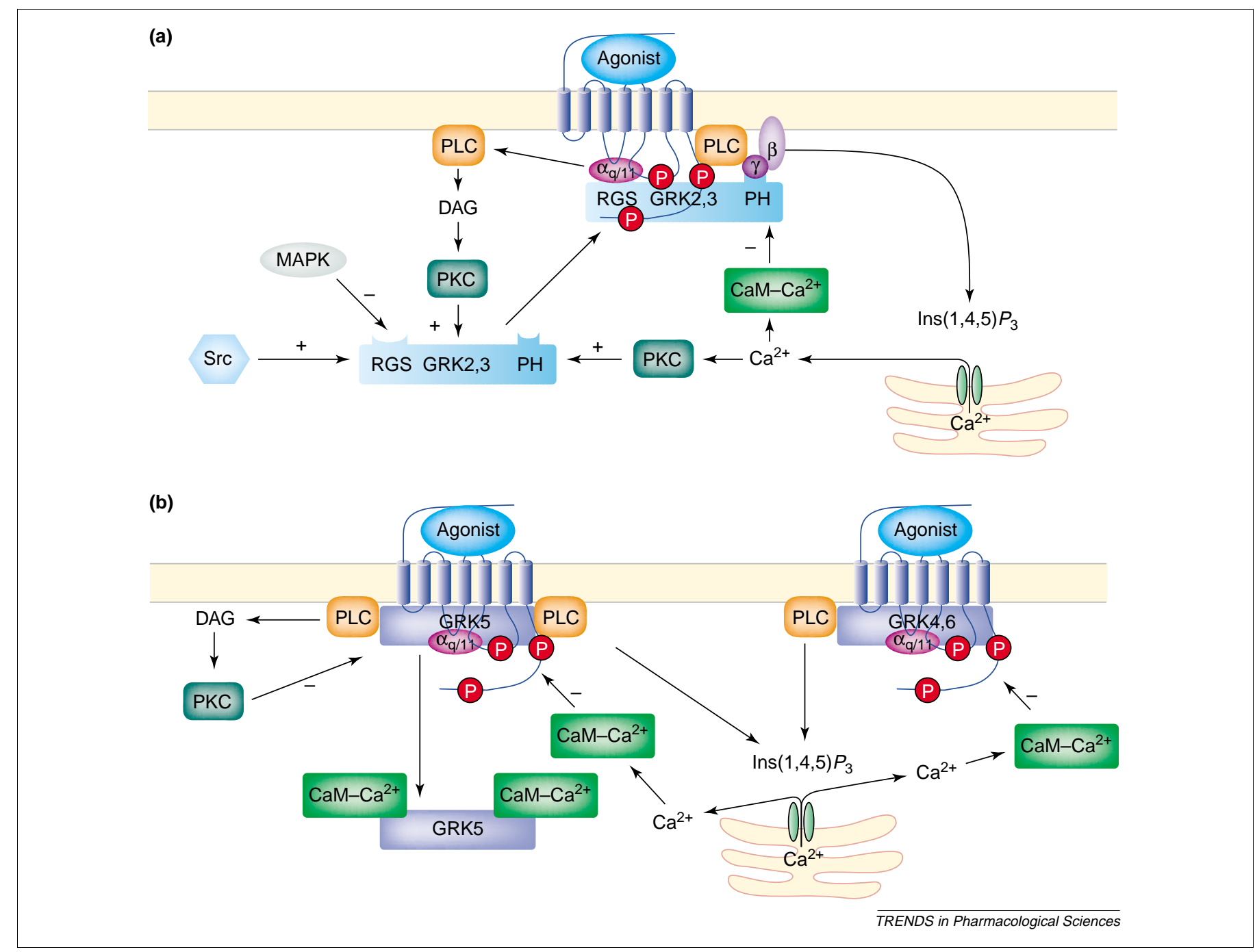

Figure 2. Models of potential G-protein-coupled receptor kinase (GRK) regulation of phospholipase C (PLC)-coupled receptors. (a) Regulation of the GRK2 and GRK3 subfamily. Both GRK2 and GRK3 are cytosolic proteins that are recruited to the plasma membrane after receptor activation. GRK2 activity is enhanced by protein kinase C (PKC) phosphorylation [58], and Src has been shown to phosphorylate GRK2 and enhance catalytic activity after receptor stimulation [59]. GRK2 is negatively regulated by $\mathrm{Ca}^{2+}$-calmodulin (CaM) and mitogen-activated protein kinase (MAPK; extracellular signal-regulated kinase), which highlights a potential negative feedback regulation of GRK2. (b) Little is known about the mechanisms that underlie recruitment of the GRK4 subfamily. However, all members of the subfamily are constitutively membrane associated (see Figure 1). In addition, all members of this group possess an N-terminal phosphatidylinositol $(4,5)$-bisphosphate binding domain, which is thought to be involved in receptor targeting and membrane association, in addition to stimulation of catalytic activity [10]. The GRK4 subfamily members are highly sensitive to $\mathrm{Ca}^{2+}-\mathrm{CaM}$ (relative to GRK2 and GRK3). The regulation of GRK5 has been studied in greatest depth, with CaM binding sites being mapped to the $\mathrm{N}$ - and C-termini [60]. Current evidence suggests that the latter is of greater functional significance [48,60]. CaM binding leads to loss of GRK5 membrane association [48]. Phosphorylation by PKC, mapped to amino acids 571-590 [26], inhibits GRK5 activity. Abbreviations: $\alpha_{q / 11}$, $\alpha$-subunit of $G_{q / 11}$ protein; $\beta \gamma$, $\beta \gamma$-subunits of $G_{q} / 11$ protein; DAG, diacylglycerol: Ins $(1,4,5) P_{3}$, inositol $(1,4,5)$-trisphosphate; $\mathrm{P}$, phosphate moiety; $\mathrm{PH}$, pleckstrin homology domain; RGS, regulator of $\mathrm{G}$-protein signalling-like domain; Src, non-receptor tyrosine kinase Src.

interfering peptides face formidable barriers of access to allow realistic clinical development.

One potential area of investigation is membrane association. Inhibition of GRK2 membrane recruitment in mice overexpressing the $\mathrm{PH}$ domain of GRK2 displayed enhanced cardiac contractility in vivo [51]. Furthermore, delipidated GRK6 has only a fraction of the catalytic activity of the palmitoylated form, highlighting the importance of correct membrane targeting [52]. All GRKs are inhibited by $\mathrm{Ca}^{2+}$-calmodulin (Table 2) but the GRK4 subfamily is more sensitive to such inhibition than the GRK2 subfamily (by up to 40 -fold). Thus, the development of drugs that target this binding region might allow selective inhibition of GRK4-6 over the GRK2 subfamily. Conversely, the recently identified differences in the potency of the RGS-like domains between GRKs 2 and 3 and GRK4-6 [53] could signal the development of drugs that target the RGS-like domains of GRK2 and GRK3 (Box 1). The pharmacological potential for both positive and negative regulation of RGS proteins to regulate G-protein activity and hence GPCR function has been reviewed recently [54]. GRK2 can be phosphorylated by ERK, resulting in substantially reduced catalytic activity [55] and degradation [56], suggesting another potential site for pharmacological intervention (Table 2).

\section{Concluding remarks}

Much new information regarding the phosphorylation and regulation of GPCRs by GRK2 and GRK3 and their role in GPCR signalling has been revealed during the past few 
years. More recent studies have started to indicate roles for GRK4, GRK5 and GRK6, both in transfected cell lines and in primary cells. However, it remains to be established whether the multiplicity of GRKs is related to the specificity or differential regulation of GPCR signalling or indeed other, yet to be defined, functions. The association of particular GRKs within signalling microdomains and their specific targeting to, and regulation of, receptor signalling, trafficking and switching is a key area of current and future investigation. Similarly, the clear evidence that GRK2 and GRK3 can act as powerful (type C) RGS proteins to suppress $\mathrm{G}_{\mathrm{q} / 11}-\mathrm{PLC}$ signalling, independently of receptor phosphorylation, needs to be investigated at the level of endogenous signalling components in vivo. Collectively, this new information offers novel targets for future pharmacological intervention.

\section{Acknowledgements}

Our work is supported by a Wellcome Trust Programme Grant (No. 062495). We would like to thank Mark Nash, Ken Young, Andrew Tobin and Eamonn Kelly (University of Bristol), for many valuable discussions.

\section{References}

1 Pierce, K.L. et al. (2002) Seven-transmembrane receptors. Nat. Rev. Mol. Cell Biol. 3, 639-650

2 Fredriksson, R. et al. (2003) The G protein-coupled receptors in the human genome form five main families. Phylogenetic analysis, paralogon groups, and fingerprints. Mol. Pharmacol. 63, 1256-1272

3 Wilson, S. et al. (1998) Orphan G protein-coupled receptors: the next generation of drug targets? Br. J. Pharmacol. 125, 1387-1392

4 Hausdorff, W.P. et al. (1990) Turning-off the signal: desensitization of $\beta$-adrenergic receptor function. FASEB J. 4, 2881-2889

5 Ferguson, S.S.G. (2001) Evolving concepts in G protein-coupled receptor endocytosis: the role in receptor desensitization and signaling. Pharmacol. Rev. 53, 1-24

6 Pierce, K.L. and Lefkowitz, R.J. (2001) Classical and new roles of $\beta$-arrestins in the regulation of G-protein-coupled receptors. Nat. Rev. Neurosci. 2, 727-733

7 Tobin, A.B. et al. (1997) Stimulus-dependent phosphorylation of G-protein-coupled receptors by casein kinase $1 \alpha$. J. Biol. Chem. 272 , 20844-20849

8 Budd, D.C.etal. (2000) Phosphorylation and regulation of a $\mathrm{G}_{\mathrm{q} / 11^{-}}$-coupled receptor by casein kinase $1 \alpha$. J. Biol. Chem. 275, 19667-19675

9 Budd, D.C. et al. (2001) Phosphorylation of the $\mathrm{G}_{\mathrm{q} / 11^{-}}$-coupled m3-muscarinic receptor is involved in receptor activation of the ERK-1/2 mitogen-activated protein kinase pathway. J. Biol. Chem. 276, 4581-4587

10 Pitcher, J.A. et al. (1998) G protein-coupled receptor kinases. Annu. Rev. Biochem. 67, 653-692

11 Erdtmann-Vourliotis, M. et al. (2001) Distribution of G protein-coupled receptor kinase (GRK) isoforms 2, 3,5 and 6 in the rat brain. Brain Res. Mol. Brain Res. 95, 129-137

12 Willars, G.B. et al. (1999) Receptor phosphorylation does not mediate cross talk between muscarinic $\mathrm{M}_{3}$ and bradykinin $\mathrm{B}_{2}$ receptors. Am. J. Physiol. 277, C859-C869

13 Blaukat, A. et al. (2001) Determination of bradykinin $\mathrm{B}_{2}$ receptor in vivo phosphorylation sites and their role in receptor function. J. Biol. Chem. 276, 40431-40440

14 Ahamed, J. and Ali, H. (2002) Distinct roles of receptor phosphorylation, G protein usage, and mitogen-activated protein kinase activation on platelet activating factor-induced leukotriene $\mathrm{C}_{4}$ generation and chemokine production. J. Biol. Chem. 277, 22685-22691

15 Pals-Rylaarsdam, R. et al. (1995) Desensitization and internalization of the $\mathrm{m} 2$ muscarinic acetylcholine receptor are mediated by independent mechanisms. J. Biol. Chem. 270, 29004-29011

16 Maestes, D.C. et al. (1999) Differential phosphorylation paradigms dictate desensitization and internalization of the $N$-formyl peptide receptor. J. Biol. Chem. 274, 29791-29795

17 Lamey, M. et al. (2002) Distinct residues in the carboxyl tail mediate agonist-induced desensitization and internalization of the human dopamine $\mathrm{D}_{1}$ receptor. J. Biol. Chem. 277, 9415-9421

18 Marchese, A. et al. (2003) The ins and outs of G protein-coupled receptor trafficking. Trends Biochem. Sci. 28, 369-376

19 Sallese, M. et al. (1997) G protein-coupled receptor kinase GRK4. Molecular analysis of the four isoforms and ultrastructural localization in spermatozoa and germinal cells. J. Biol. Chem. 272, 10188-10195

20 Virlon, B. et al. (1998) Rat G protein-coupled receptor kinase GRK4: identification, functional expression, and differential tissue distribution of two splice variants. Endocrinology 139, 2784-2795

21 Sallese, M. et al. (2000) The G protein-coupled receptor kinase GRK4 mediates homologous desensitization of metabotropic glutamate receptor 1. FASEB J. 14, 2569-2580

22 Carman, C.V. et al. (1999) Selective regulation of $\mathrm{G}_{\mathrm{q} / 11} \alpha$ by an RGS domain in the G protein-coupled receptor kinase GRK2. J. Biol. Chem. 274, 34483-34492

23 Sallese, M. et al. (2000) Selective regulation of $\mathrm{G}_{\mathrm{q}}$ signaling by $\mathrm{G}$ protein-coupled receptor kinase 2: direct interaction of kinase $N$-terminus with activated $\mathrm{G} \alpha_{\mathrm{q}}$. Mol. Pharmacol. 57, 826-831

24 Dhami, G.K. et al. (2002) Phosphorylation-independent regulation of metabotropic glutamate receptor signaling by $\mathrm{G}$ protein-coupled receptor kinase 2. J. Biol. Chem. 277, 25266-25272

25 Eichmann, T. et al. (2003) The amino-terminal domain of G proteincoupled receptor kinase 2 is a regulatory G $\beta \gamma$ binding site. J. Biol. Chem. 278, 8052-8057

26 Pronin, A.N. et al. (1998) Structure-function analysis of G proteincoupled receptor kinase-5. Role of the carboxyl terminus in kinase regulation. J. Biol. Chem. 273, 31510-31518

27 Premont, R.T. et al. (1999) The GRK4 subfamily of G protein-coupled receptor kinases. Alternative splicing, gene organization, and sequence conservation. J. Biol. Chem. 274, 29381-29389

28 Iacovelli, L. et al. (2003) Role of G protein-coupled receptor kinase 4 and $\beta$-arrestin 1 in agonist-stimulated metabotropic glutamate receptor 1 internalization and activation of mitogen-activated protein kinases. J. Biol. Chem. 278, 12433-12442

29 Watanabe, H. et al. (2002) Desensitization of human $\mathrm{D}_{1}$ dopamine receptors by $\mathrm{G}$ protein-coupled receptor kinase 4. Kidney Int. 62, 790-798

30 Perroy, J. et al. (2003) Phosphorylation-independent desensitization of $\mathrm{GABA}_{\mathrm{B}}$ receptors by GRK4. EMBO J. 22, 3816-3824

31 Felder, R.A. et al. (2002) G protein-coupled receptor kinase 4 gene variants in human essential hypertension. Proc. Natl. Acad. Sci. U.S.A. 99, 3872-3877

32 Shetzline, M.A. et al. (2002) Vasoactive intestinal polypeptide type-1 receptor regulation: Desensitization, phosphorylation and sequestration. J. Biol. Chem. 277, 25519-25526

33 Tiruppathi, C. et al. (2000) G protein-coupled receptor kinase-5 regulates thrombin-activated signaling in endothelial cells. Proc. Natl. Acad. Sci. U.S.A. 97, 7440-7445

34 Nagayama, Y. et al. (1996) Involvement of G protein-coupled receptor kinase 5 in homologous desensitization of the thyrotropin receptor. J. Biol. Chem. 271, 10143-10148

35 Gainetdinov, R.R. et al. (1999) Muscarinic supersensitivity and impaired receptor desensitization in $\mathrm{G}$ protein-coupled receptor kinase 5-deficient mice. Neuron 24, 1029-1036

36 Lazari, M.F. et al. (1999) Role of G protein-coupled receptor kinases on the agonist-induced phosphorylation and internalization of the follitropin receptor. Mol. Endocrinol. 13, 866-878

37 Aiyar, N. et al. (2000) Involvement of G protein-coupled receptor kinase-6 in desensitization of CGRP receptors. Eur. J. Pharmacol. 403, $1-7$

38 Willets, J.M. et al. (2001) GRK3 and GRK6 utilize different pathways to desensitize the endogenous $\mathrm{M}_{3}$ muscarinic acetylcholine receptor in human SH-SY5Y cells. Mol. Pharmacol. 60, 321-330

39 Willets, J.M. et al. (2002) Endogenous G protein-coupled receptor kinase 6 regulates $M_{3}$ muscarinic acetylcholine receptor phosphorylation and desensitization in human SH-SY5Y neuroblastoma cells. J. Biol. Chem. 277, 15523-15529

40 Ghadessy, R.S. et al. (2003) G protein-coupled receptor kinase 6 
(GRK6) selectively regulates endogenous secretin receptor responsiveness in NG108-15 cells. Br. J. Pharmacol. 138, 660-670

41 Willets, J.M. et al. (1999) Selective reduction in $\mathrm{A}_{2}$ adenosine receptor desensitization following antisense-induced suppression of $\mathrm{G}$ proteincoupled receptor kinase 2 expression. J. Neurochem. 73, 1781-1789

42 Simon, V. et al. (2003) Endogenous G protein-coupled receptor kinase 6 triggers homologous $\beta$-adrenergic receptor desensitization in primary uterine smooth muscle cells. Endocrinology 144, 3058-3066

43 Fong, A.M. et al. (2002) Defective lymphocyte chemotaxis in $\beta$-arrestin-2- and GRK6-deficient mice. Proc. Natl. Acad. Sci. U.S.A. 99, 7478-7483

44 Gainetdinov, R.R. et al. (2003) Dopaminergic supersensitivity in G protein-coupled receptor kinase 6-deficient mice. Neuron 38, 291-303

45 Kohout, T.A. and Lefkowitz, R.J. (2003) Regulation of G proteincoupled receptor kinases and arrestins during receptor desensitization. Mol. Pharmacol. 63, 9-18

46 Penn, R.P. et al. (2000) Regulation of G protein-coupled receptor kinases. Trends Cardiovasc. Med. 10, 81-89

47 Willars, G.B. et al. (1998) Differential regulation of muscarinic acetylcholine receptor-sensitive polyphosphoinositide pools and consequences for signaling in human neuroblastoma cells. J. Biol. Chem. $273,5037-5046$

48 Pronin, A.N. et al. (1997) Regulation of G protein-coupled receptor kinases by calmodulin and localization of the calmodulin binding domain. J. Biol. Chem. 272, 18273-18280

49 Budd, D.C. et al. (1999) Cross-talk between m3-muscarinic and $\beta_{2}$-adrenergic receptors at the level of receptor phosphorylation and desensitization. Mol. Pharmacol. 56, 813-823

50 Pollok-Kopp, B. et al. (2003) Analysis of ligand-stimulated CC chemokine receptor 5 (CCR5) phosphorylation in intact cells using phosphosite-specific antibodies. J. Biol. Chem. 278, 2190-2198

51 Koch, W.J. et al. (1995) Cardiac function in mice overexpressing the $\beta$-adrenergic kinase or a $\beta$-ARK inhibitor. Science $268,1350-1353$

52 Loudon, R.P. and Benovic, J.L. (1997) Altered activity of palmitoylation-deficient and isoprenylated forms of the $\mathrm{G}$ protein-coupled receptor kinase GRK6. J. Biol. Chem. 272, 27422-27427

53 Sterne-Marr, R. et al. (2003) G protein-coupled receptor kinase $2 / \mathrm{G} \alpha_{\mathrm{q} / 11}$ interaction: a novel surface on a regulator of $G$ protein signaling homology domain for G $\alpha$ subunits. J. Biol. Chem. 278, 6050-6058

54 Neubig, R.R. and Siderovski, D.P. (2002) Regulators of G-protein signaling as new central nervous system drug targets. Nat. Rev. Drug Discov. 1, 187-197

55 Pitcher, J.A. et al. (1999) Feedback inhibition of G protein-coupled receptor kinase 2 (GRK2) activity by extracellular signal-regulated kinases. J. Biol. Chem. 274, 34531-34534

56 Elorza, A. et al. (2003) MAPK-dependent degradation of G proteincoupled receptor kinase 2. J. Biol. Chem. 278, 29164-29173

57 Lodowski, D.T. et al. (2003) Keeping G proteins at bay: a complex between G protein-coupled receptor kinase 2 and G $\beta \gamma$. Science 300, $1256-1262$
58 Winstel, R. et al. (1996) Protein kinase cross-talk: membrane targeting of the $\beta$-adrenergic receptor kinase by protein kinase C. Proc. Natl. Acad. Sci. U.S.A. 93, 2105-2109

59 Fan, G. et al. (2001) c-Src tyrosine kinase binds the $\beta_{2}$-adrenergic receptor via phosphor-Tyr-350, phosphorylates G protein-linked receptor kinase 2 , and mediates agonist-induced receptor desensitization. J. Biol. Chem. 276, 13240-13247

60 Levay, K. et al. (1998) Localization of the sites for $\mathrm{Ca}^{2+}$-binding proteins on the G protein-coupled receptor kinases. Biochemistry 37, 13650-13659

61 Gainetdinov, R.R. et al. (2000) Reply: specificity of G-protein-coupled receptor kinases. Trends Pharmacol. Sci. 21, 366-367

62 Loudon, R.P. and Benovic, J.L. (1996) Differentially regulated expression of the G-protein-coupled receptor kinases, $\beta A R K$ and GRK6, during myelomonocytic cell development in vitro. Blood 88, $4547-4557$

63 Schleicher, S. et al. (1993) A $\beta$-adrenergic receptor kinase is involved in olfactory signal termination. Proc. Natl. Acad. Sci. U.S.A. 90, $1420-1424$

64 Premont, R.T. et al. (1996) Characterization of the G protein-coupled receptor kinase GRK4: Identification of four splice variants. J. Biol. Chem. 271, 6403-6410

65 Lombardi, M.S. et al. (1999) Decreased expression and activity of G Protein-coupled receptor kinases in peripheral blood mononuclear cells of patients with rheumatoid arthritis. FASEB J. 13, 715-725

66 Freeman, J.L.R. et al. (2000) $\alpha$-Actinin is a potent regulator of G protein-coupled receptor kinase activity and substrate specificity in vitro. FEBS Lett. 473, 280-284

67 Pitcher, J.A. et al. (1992) Role of $\beta \gamma$ subunits of $G$ proteins in targeting the $\beta$-adrenergic receptor kinase to membrane-bound receptors. Science 257, 1264-1267

68 Pronin, A.N. and Benovic, J.L. (1997) Regulation of G protein-coupled receptor kinase 5 by protein kinase C. J. Biol. Chem. 272, 3806-3812

69 Krasel, C. et al. (2001) Phosphorylation of GRK2 by Protein kinase C abolishes its inhibition by calmodulin. J. Biol. Chem. 276, 1911-1915

70 Penella, P. et al. (2001) $\beta$-arrestin- and c-Src-dependent degradation of G protein-coupled receptor kinase 2. EMBO J. 20, 5129-5138

71 Diviani, D. et al. (1996) Effect of different G protein-coupled receptor kinases on phosphorylation and desensitization of the $\alpha_{1 \mathrm{~B}}$-adrenergic receptor. J. Biol. Chem. 271, 5049-5058

72 Freedman, N.J. et al. (1997) Phosphorylation and desensitization of human endothelin A and B receptors: evidence for G protein-coupled receptor kinase specificity. J. Biol. Chem. 272, 17734-17743

73 Iacovelli, L. et al. (1999) Selective regulation of G protein-coupled receptor-mediated signaling by $\mathrm{G}$ protein-coupled receptor kinase 2 in FRTL-5 cells: analysis of thyrotropin, $\alpha_{1 \mathrm{~B}}$-adrenergic, and $\mathrm{A}_{1}$ adenosine receptor-mediated responses. Mol. Pharmacol. 56, 316-324

74 Dicker, F. et al. (1999) Phosphorylation-independent inhibition of parathyroid hormone receptor signaling by $\mathrm{G}$ protein-coupled receptor kinases. Proc. Natl. Acad. Sci. U.S.A. 96, 5476-5481

\section{Recently published books of interest}

- Immunosuppressant Analogs in Neuroprotection Edited by Cesario V. Borlongan, Ole Isacson and Paul R. Sanberg, Humana Press, 2003. \$150.00 (416 pages) ISBN 0896039447

- In Vitro Neurotoxicology: Principles and Challenges Edited by Evelyn Tiffany-Castiglioni, Humana Press, 2003. \$125.00 (350 pages) ISBN 1588290476

- Molecular Biology of Drug Addiction Edited by Rafael Maldonado, Humana Press, 2003. \$135.00 (360 pages) ISBN 1588290603

- Opioid Research: Methods and Protocols Edited by Zhizhong Z. Pan, Humana Press, 2003. \$125.00 (308 pages) ISBN 158829059 X

- G Protein Signaling: Methods and Protocols Edited by Alan V. Smrcka, Humana Press, 2003. \$79.50 (264 pages) ISBN 1592594301

- Suicide Gene Therapy: Methods and Reviews Edited by Caroline J. Springer, Humana Press, 2003. \$130.00 (560 pages) ISBN 1592594298

- The Macrophage as a Therapeutic Target Edited by Siamon Gordon, Springer, 2003. £219.00 (496 pages) ISBN 3540442502 